\title{
A randomized controlled trial on Aspirin and complex regional pain syndrome after radius fractures
}

\author{
Amir Sobhani Eraghi (1), Amir Khazanchin (2), Nima Hosseinzadeh (1,2), Alireza \\ Pahlevansabagh (1)
}

(1) Department of Orthopaedics Surgery, Rasoul Akram Hospital, Iran University of Medical Sciences, Tehran, Iran; (2). Bone and Joint Reconstruction Research Center, Shafa Orthopedic Hospital, Iran University of Medical Sciences, Tehran, Iran

This article is distributed under the terms of the Creative Commons Attribution Noncommercial License (CC BY-NC 4.0) which permits any noncommercial use, distribution, and reproduction in any medium, provided the original author(s) and source are credited.

\begin{abstract}
Complex regional pain syndrome (CRPS) is often diagnosed in patients who are recovered with surgery or injury. CRPS is usually diagnosed in patients recovering from distal radius fractures. The aim of study was the effects of aspirin in prevention of the complex regional pain syndrome (CRPS) following a fracture of distal radius. In a double-blind, randomized controlled trial, 91 patients with unilateral extra-articular distal radius fractures were randomly allocated to receive either placebo (PLA) or $500 \mathrm{mg}$ of aspirin (ASA) daily for 7 days. The effect of aspirin on the occurrence of CRPS was evaluated. The patients were assessed clinically and radiographically in the second, fourth and twelfth weeks by a physician who was unaware of the treatment allocation. Ninety-one patients (ASA, n=44; PLA, n=47) were enrolled in the study. The prevalence of CRPS in all patients was $16.5 \%$. The prevalence of CRPS in the aspirin group was lower $(13.6 \%)$ than the placebo group $(19.1 \%)$, but this difference was not statistically significant. The only significant difference was the lower rate of regional osteoporosis seen in the radiographs of aspirin group. Mean age was significantly higher in the patients with CRPS. Also, comminuted distal radius fractures (A3-type) were significantly more common in the patients with CRPS. Administration of aspirin in patients with a distal radius fracture was associated with a lower incidence of CRPS, but, not statistically significant. Further investigations needs to be done with a larger sample size, longer follow-up period and multicenter design.
\end{abstract}

Key Words: Distal radius fractures; Complex Regional Pain Syndrome; Aspirin; Reflex Sympathetic Dystrophy.

Eur J Transl Myol 30 (1): xx1-xx8, 2020

Distal radius fractures are the most common fractures that orthopedic surgeons face in their traumatic patients. They are responsible for up to $18 \%$ of all fractures in the elderly population. Most patients heal uneventfully, but up to $37 \%$ of these fractures may complicate with a chronic neurological condition called Complex Regional Pain Syndrome (CRPS). ${ }^{1,2}$ CRPS, also known as Reflex Sympathetic Dystrophy (RSD), is characterized by severe pain along with sensory, autonomic, motor and trophic impairment - ranging from mild and self-limiting to chronic disease- ${ }^{3}$ resulting in dysfunction and disability to do daily living activities. ${ }^{4}$ The pain experienced is proportional to the extent of tissue damage and is beyond the normal expected time for tissue repair. The pathophysiology is multifactorial and involves pain regulation in both the sympathetic and central nervous systems, with likely genetic, inflammatory and psychological contributions. ${ }^{5}$ There are several theories for its pathophysiology, including an exaggerated inflammatory response to a trauma, altered sympathetic nervous system function, catecholamines, autoimmunity, genetic and psychological factors. ${ }^{4}$ Moreover, there is a plethora of medications used to prevent or treat CRPS, mostly because of the poorly understood pathophysiology. ${ }^{6}$

It has been hypothesized that the exaggerated inflammatory response to a trauma has an important role in the pathophysiology of CRPS. Clinical presentation of the acute phase of CRPS, i.e., the affected limb reveals pain, edema, erythema, increased temperature and impaired function, supports this hypothesis. ${ }^{4,7}$ Nonsteroidal anti-inflammatory drugs (NSAIDs), used to 


\section{Complex regional pain syndrome}

Eur J Transl Myol 30 (1): xx1-xx8, 2020

treat the inflammatory symptoms of CRPS and to relief pain, act by inhibiting cyclooxygenase and preventing the synthesis of prostaglandins, which mediate inflammation and hyperalgesia. Studies have demonstrated the role of spinal cyclooxygenase and its effect on hyperalgesia and allodynia. ${ }^{8-11}$ However, there is a renewed interest in studying NSAIDs.

Aspirin, also known as acetylsalicylic acid (ASA), is a medication used to treat pain, fever, or inflammation. The anti-inflammatory effects of ASA are mediated by the inhibition of cyclooxygenase enzymes. Aspirin works by blocking the production of prostaglandins, the on-off switch in cells that regulate pain and inflammation, among other things. That's why aspirin stops mild inflammation and pain. ${ }^{12,13}$ Given the multifactorial nature and complex pathophysiology of CRPS, the number of Randomized Controlled Trials (RCTs) assessing the efficacy of pharmacologic agents for its prevention and treatment are few, small in scale, noncontrolled, or reported in poster form at meetings. ${ }^{10}$
In this RCT study we aimed to evaluate the effects of aspirin in prevention of the CRPS following a fracture of distal radius.

\section{Materials and Methods}

\section{Study design}

This double-blind, randomized controlled trial was conducted from August 2016 to September 2017, at Rasoul Akram Hospital, in Tehran, Iran. The authors adhered to the Consolidated Standards of Reporting Trials (CONSORT) guidelines.

Level of Evidence: Level I, Therapeutic study, randomized controlled trial (RCT).

\section{Patient selection}

The method of sampling was census, in such a way that patients referred or presented to the emergency department of the hospital, with an acute distal radius fracture, were entered into the study during 24 months. Inclusion criteria includes all patients with eighteen years old and over with a closed, unilateral, extra-articular

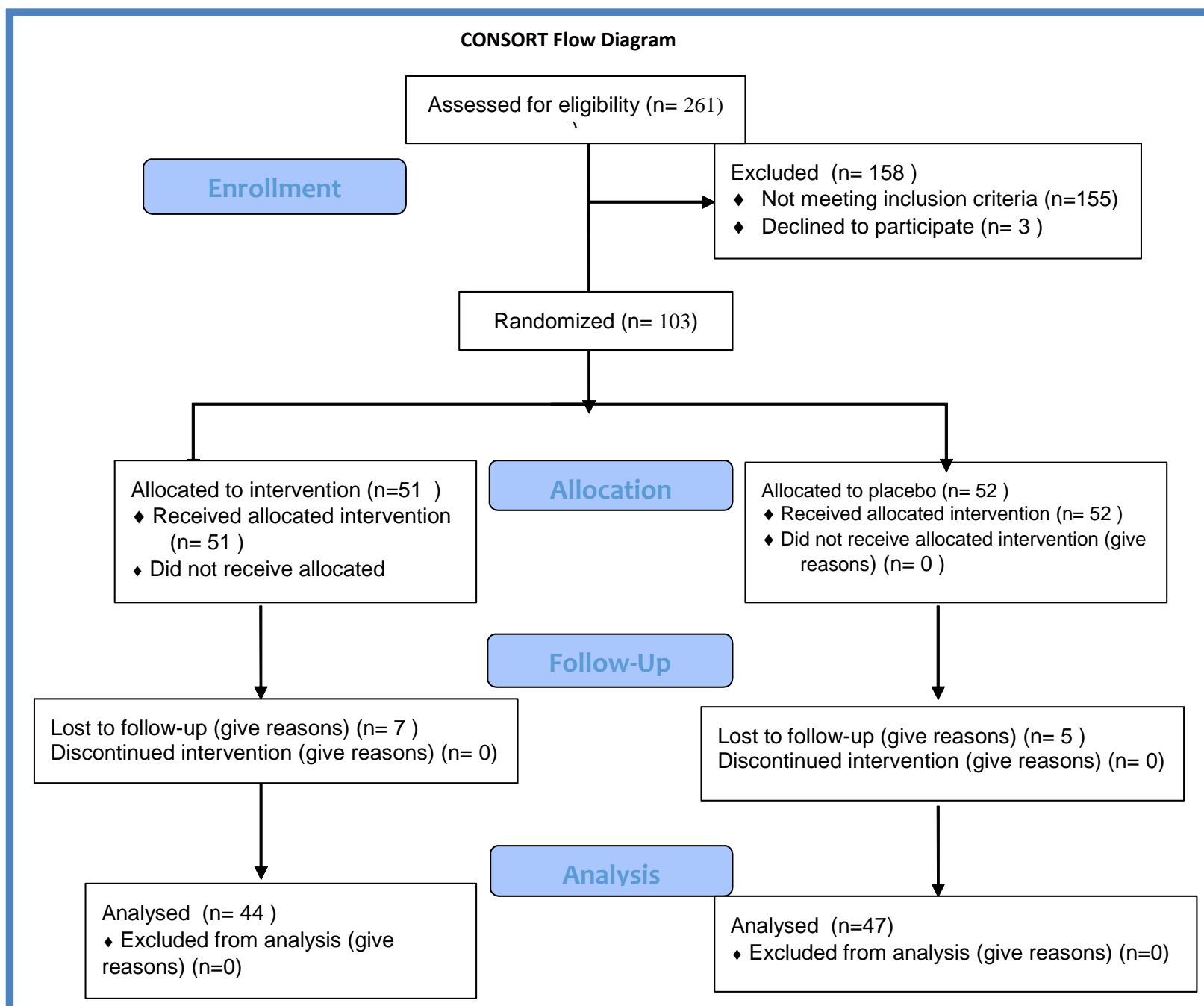

Fig. 1 CONSORT flow diagram 
distal radius fracture. Exclusion criteria were a history of taking medications for CRPS treatment (antidepressants, anticonvulsants, corticosteroids and vitamin C); previous wrist or hand fracture on the same side; articular displacement requiring open reduction; open fractures; neurovascular injury; fractures with high energy mechanism, multiple trauma-injured patients; multiple fractures at different places; and contraindication to take aspirin and acetaminophen. From a total of 261 patients, 179 were studied and all fractures were assessed individually. Eventually, due to some data loss and patients being excluded from the study, 91patients were evaluated (Figure 1).

\section{Study protocol}

The protocol was initiated in the emergency department after the enrolled persons signed an informed consent. Patients were randomly allocated to receive a box with 7 capsules of either $500 \mathrm{mg}$ aspirin or placebo which should be taken one capsule daily. All boxes and capsules had been made by a pharmacist with the same appearance and taste. Randomization was performed by a pharmacist using a table of random numbers. The study was doubleblind and all participants and physicians were unaware of the treatment allocation and the pharmacist was the only person accessing to the codes until the end of the trial. All patients in the study received $1 \mathrm{gr}$ intravenous of Apotel every 6 hours at the hospital and one tablet of acetaminophen $500 \mathrm{mg}$ every 6 hours for a week for pain management.

\section{Surgical technique}

Bier's block anesthesia was done for all patients. Reduction was applied by the "Agee maneuver" with traction and flexion. Then, percutaneous pinning was performed with two pins from radial styloid and, in some patients, an extra pin from the dorsal ulnar aspect of the radius to the volar aspect, for further stability of the reduction. After the surgery, long arm cast was applied for all patients. Four weeks after the surgery, cast was changed to the below elbow cast and at the sixth week, cast and pins were removed.

\section{Follow-up}

Demographic characteristics, including age, gender, side of the fracture, hand dominance, and fracture type (according to the AO/OTA classification) were recorded at the time of presentation to the emergency department (Table 1). Other information about the symptoms and signs of the CRPS and radiographic evaluation were recorded during the follow-up period. To improve patient adherence, a follow-up phone call was made on the fourth or fifth day of the prescription course. The patients were assessed clinically and radiographically in the second week, the fourth week (the cast was shortened), the sixth week (pins and cast were removed) and the twelfth week by a physician who was unaware of the treatment allocation. Radiographic evaluation including standard postero-anterior and lateral radiographs of the injured wrist as well as one set of radiographs of the contralateral wrist, for comparison, was performed at each visit.

\begin{tabular}{|c|c|c|c|c|}
\hline \multicolumn{5}{|c|}{ Table 1. Demographic characteristics of the patients } \\
\hline \multicolumn{2}{|l|}{ Characteristic } & $\begin{array}{l}\text { Aspirin } \\
\mathrm{N}=\mathbf{4 4}\end{array}$ & $\begin{array}{l}\text { Placebo } \\
\text { N=47 }\end{array}$ & p value \\
\hline \multicolumn{2}{|l|}{ Age $($ Mean \pm SD) } & $50.86 \pm 13.54$ & $52.40 \pm 14.55$ & 0.603 \\
\hline \multirow[t]{2}{*}{ Gender } & Male & $25(57 \%)$ & $33(70 \%)$ & \multirow[t]{2}{*}{0.184} \\
\hline & Female & $19(43 \%)$ & $14(30 \%)$ & \\
\hline \multirow[t]{2}{*}{ Hand dominance } & Left & $5(11 \%)$ & $9(20 \%)$ & \multirow[t]{2}{*}{0.304} \\
\hline & Right & $39(89 \%)$ & $38(80 \%)$ & \\
\hline \multirow[t]{2}{*}{ Fracture side } & Left & $24(55 \%)$ & $21(45 \%)$ & \multirow[t]{2}{*}{0.347} \\
\hline & Right & $20(45 \%)$ & $26(55 \%)$ & \\
\hline \multirow[t]{2}{*}{ Fracture type } & $\mathbf{A 2}$ & $23(52 \%)$ & $25(53 \%)$ & \multirow[t]{2}{*}{$\mathbf{0 . 9 3}$} \\
\hline & $\mathbf{A 3}$ & $21(48 \%)$ & $22(47 \%)$ & \\
\hline
\end{tabular}


Radiographic findings including patchy, subchondral, or subperiosteal osteoporosis were diagnosed by a radiologist. The follow-up period ended in December 2017. CRPS was clinically assessed with the use of the "Budapest criteria", which has an acceptable sensitivity and specificity. ${ }^{3,14,15}$ If the diagnosis of CRPS was made, the patient was treated by reassurance, analgesia, and careful physical therapy avoiding exacerbation of pain.

\section{Ethical consideration}

Ethical considerations were observed in this study with ethics code of IR.IUMS.FMD.REC 1396.9311242007 and registered in Iranian Registry of Clinical Trials as: (IRCT20180116038391N1) bestowed by Committee of Ethics in Medical Research at Iran University of Medical Sciences. The qualified persons were identified for participation, informed written consent was obtained from each participant, the research goals and procedures were explained, principles of information confidentiality and anonymity were observed, and finally, the participants could leave the study at any stage.
A written Informed consent was obtained from all patients contributed in the study.

\section{Statistical analysis}

Analysis was performed using SPSS ver.18 (SPSS Inc., Chicago, USA). Main null hypothesis: there were no significant differences in the incidence of CRPS between the aspirin group and placebo group. For analysis of the difference between groups, $\chi 2$-test and independentsamples t-test were used to compare proportions and continuous variables, respectively. A p-value $<0.05$ was considered statistically significant.

\section{Results}

During a 14-month period, a total number of 103 patients with unilateral, extra- articular distal radius fractures participated in the study. Fifty-one patients were randomized to receive aspirin, and fifty-two received placebo. Twelve patients did not complete the follow-up period (one patient was expired because of cerebrovascular accident, 6 patients were from other

Table 2. Prevalence of positive signs and symptoms between the two groups

\begin{tabular}{|c|c|c|c|c|}
\hline Criterion & $\begin{array}{l}\text { CRPS } \\
N=15\end{array}$ & $\begin{array}{l}\text { Aspirin } \\
\mathrm{N}=44\end{array}$ & $\begin{array}{l}\text { Placebo } \\
\text { N=47 }\end{array}$ & p value \\
\hline \multicolumn{5}{|l|}{ Symptoms } \\
\hline Sensory & $6(40 \%)$ & $5(11 \%)$ & $4(9 \%)$ & $\mathbf{0 . 7 3 4}$ \\
\hline vasomotor & $10(67 \%)$ & $7(16 \%)$ & $9(19 \%)$ & 0.685 \\
\hline Sudomotor/edema & $15(100 \%)$ & $18(41 \%)$ & $15(32 \%)$ & 0.372 \\
\hline Motor/trophic & $15(100 \%)$ & $17(39 \%)$ & $19(40 \%)$ & 0.862 \\
\hline \multicolumn{5}{|l|}{ Signs } \\
\hline Sensory & $10(67 \%)$ & $7(16 \%)$ & $9(19 \%)$ & 0.685 \\
\hline vasomotor & $5(33 \%)$ & $6(14 \%)$ & $9(19 \%)$ & 0.479 \\
\hline Sudomotor/edema & $7(47 \%)$ & $14(32 \%)$ & $13(28 \%)$ & 0.664 \\
\hline Motor/trophic & $10(67 \%)$ & $13(29 \%)$ & $13(28 \%)$ & 0.842 \\
\hline CRPS & & $6(13.6 \%)$ & $9(19.1 \%)$ & 0.479 \\
\hline \multicolumn{5}{|c|}{ Time of diagnosis (CRPS) } \\
\hline 4 weeks & & $1(17 \%)$ & $2(22 \%)$ & 1.00 \\
\hline 6 weeks & & $5(83 \%)$ & $7(78 \%)$ & \\
\hline Radiographic changes & & $7(16 \%)$ & $16(34 \%)$ & $0.047^{*}$ \\
\hline
\end{tabular}


Table 3. Difference of characteristics between patients with or without CRPS

\begin{tabular}{|c|c|c|c|c|}
\hline \multicolumn{2}{|l|}{ Characteristic } & $\begin{array}{l}\text { CRPS } \\
\text { N=15 }\end{array}$ & $\begin{array}{l}\text { No CRPS } \\
\text { N=76 }\end{array}$ & p value \\
\hline \multicolumn{2}{|l|}{ Age $($ Mean \pm SD $)$} & $60.27 \pm 9.35$ & $49.96 \pm 14.19$ & $\mathrm{0.008}^{*}$ \\
\hline \multirow[t]{2}{*}{ Gender } & Male & $8(53 \%)$ & $50(66 \%)$ & \multirow[t]{2}{*}{0.359} \\
\hline & Female & $7(47 \%)$ & $26(34 \%)$ & \\
\hline \multirow[t]{2}{*}{ Fracture type } & A2 & $4(27 \%)$ & $44(58 \%)$ & \multirow[t]{2}{*}{$0.027^{*}$} \\
\hline & A3 & $11(73 \%)$ & $32(42 \%)$ & \\
\hline
\end{tabular}

states and 5 patients did not continue follow-up after removing cast and pins at the sixth week). In total, 91 patients remained for final assessment that forty-four of them received aspirin (as aspirin group), and 47 received placebo (placebo group).

There were no significant differences between two groups in terms of demographic characteristics including age, gender, hand dominance, fracture side and fracture type (Table1).

The prevalence of CRPS in all patients was $16.5 \%$ (15 patients out of 91 patients). Although, analysis of the two groups showed that the prevalence of CRPS in the aspirin group was lower $(13.6 \%, 6$ of 44 patients) than the placebo group (19.1\%, 9 of 47 patients), this difference was not statistically significant $(\mathrm{p}=0.479)$. Information about the prevalence of the symptoms and signs in the patients with CRPS and patients who received aspirin or placebo are shown in Table 2.The only significant difference was the lower rate of regional osteoporosis seen in the radiographs of the aspirin group $(\mathrm{P}=0.047)$, suggesting a significant relationship between the use of aspirin and the reduction of radiographic evidence for osteoporosis. All fractures healed successfully after 12 weeks. In most patients, CRPS was diagnosed at 6 weeks after the fracture and diagnosis time was not significantly different between the two groups.

There was no difference regarding to the gender between the patients with or without CRPS. Mean age was significantly higher in patients with CRPS $(\mathrm{P}=0.008)$. Also, comminuted distal radius fractures (A3-type) were significantly more common in the patients with CRPS (P $=0.027$ ). Data are shown in Table 3 .

Only 7 patients had pin tract infections (4 form aspirin group and 3 from placebo group, $\mathrm{P}=0.708$ ), which all of them were grade I and II and treated without complications.

\section{Discussion}

The complex regional pain syndrome is a nonspecific condition with variable symptoms and uncertain values. ${ }^{16}$
It was usually diagnosed in the recovery period after distal radius fracture. ${ }^{17}$ While acute CRPS sometimes improves spontaneously or with aggressive physical therapy, CRPS present for a period of one year or greater seldom spontaneously resolves 33 , and worsens in many patients from years 1 to 8 after onset. ${ }^{18}$

This study demonstrated that administration of aspirin in the patients with a distal radius fracture was associated with a lower frequency of CRPS compared with placebo group, but the difference was not statistically significant. Furthermore, no significant difference was found between the two groups in demographic data, fracture specifications and CRPS. The only significant difference was the lower rate of regional osteoporosis seen in the radiographs of the aspirin group. Moreover, CRPS was significantly more frequent in older patients and those with comminuted fractures.

The overall incidence of CRPS in the present study was $13.6 \%$ in aspirin group and $19.1 \%$ in the placebo group. In the study of Zollinger et al. ${ }^{19}$ and the studies of Atkins et al. ${ }^{20}$, CRPS incidence in the placebo group was higher (22\%, $25 \%$ and $37 \%$, respectively). This difference might be due to the different criteria of our study. We diagnosed CRPS based on the "Budapest criteria", but in the mentioned studies, different criteria have been used. Additionally, our study included only those patients who had an extra-articular fracture which was treated with percutaneous pinning.

Mean age was significantly higher in our patients with CRPS. This finding is consistent with those of Demir et al. $^{21}$ and Roh et al. ${ }^{22}$ Although Ortiz-Romero et al. ${ }^{23}$ found that patients under 60 years of age have an elevated risk of developing CRPS that might be due to highenergy fractures in these group of patients

Demir et al. and Roh et al. showed that female gender has been considered a risk factor for CRPS. ${ }^{21,} 2,24$ Also, Young-Hoon Jo et al. stated that risk factors that were significantly associated with CRPS-1 incidence included female gender, open reduction and open fracture. ${ }^{25}$ In Chung et al. study, ${ }^{26}$ sex hormones, such as estrogens, are 
of more interest with regard to CRPS, due to its high incidence in women and at postmenopausal age. However, we found no significant sex difference between the patients with or without CRPS.

Our findings showed that comminuted distal radius fractures were significantly more common in patients with CRPS. This finding could be attributed to more inflammation and injury to the bone and surrounding tissues due to transferring a higher energy to them. This relationship also has been found in other studies. ${ }^{22,27,28}$ According to a study by Goh et al, after a distal radius fracture, radiographic features of bone loss and demineralization could be seen, either as a result of disuse or CRPS progression, leading to nociceptive bone pain, osteopenia and osteoporosis. ${ }^{4}$ Interestingly, based on the study of Chung et al., osteoporosis has been considered a consequence of CRPS rather than a risk factor. ${ }^{26}$ Other results of cellular and animal studies demonstrated that aspirin possesses bone protective effects by promoting the survival of osteoblast precursor stem cells and differentiation of osteoblast, ${ }^{29-32}$ but there are studies with opposit results. ${ }^{33-35}$ The pharmacodynamics behavior of aspirin in the treatment of fracture or the prevention of CRPS is not fully understood and there is still controversy due to inconclusive data and lack of evidence. Nevertheless, our study demonstrated that regional osteoporosis in plan radiographs in aspirin group was significantly lower than the other group.

Administration of aspirin in patients with a distal radius fracture was associated with a lower frequency of CRPS than placebo group, but the difference was not statistically significant. Several mechanisms could be considered to explain these findings.

Atkins et al. highlighted that cytokine levels are higher in CRPS-affected limbs than in the contra lateral limb or control patients. ${ }^{20}$ The acute phase of CRPS is characterized by classical signs and symptoms of inflammation. This suggests that CRPS is an exaggerated local inflammatory response to injury. ${ }^{36}$ Therefore, aspirin with its anti-inflammatory effect might help the body to overcome this complication.

CRPS seems to be a local form of the systemic free radical disease. In one of the studies of van der Laan et al., ${ }^{37}$ severely thickened basal membrane layers of the capillaries, compatible with overexposure to free radicals, have been showed in amputated human specimens with CRPS.

Additionally, van der Laan et al. reported that in animals, induction of free-radical formation in one hind-limb of awaked rats mimics the acute signs and symptoms of CRPS. ${ }^{38}$ These findings are supported by the evidence from Atkins et al. that vitamin $\mathrm{C}$ is an effective prophylaxis against post-traumatic CRPS as a free radical scavenger. ${ }^{39}$ Koseoglus et al. demonstrated that a lowdose aspirin supplementation (300 mg. daily for 10 days) in a short time period significantly increases total antioxidant activity. ${ }^{40}$ Demirci et al. ${ }^{41}$ also also found that in the study of the antioxidant role of aspirin in the serum of rats, aspirin improved the relaxation function and antioxidant capacity and decreased the oxidant status. Therefore, aspirin may play a role in improving the general anti-oxidative potency of the blood, so reducing the effect of oxygen free radicals in the CRPS process as shown in studies conducted by Podhaisky, ${ }^{42}$ Ristimae et al. $^{43}$ and other studies ${ }^{44,45}$

Limitations of our study, i.e. the relatively small study population, the recruitement from only one teaching hospital and the short follow-up period, do not allow generalization of our research findings.

Positive points of the study are its prospective design and strict criteria for patient selection that decrease effects of confounding variables. Furthermore, it is the first study to evaluate the effects of aspirin in prevention of CRPS, not as a treatment.

In conclusion, although there is no definite successful method for prevention or treatment of CRPS to date, this study adds substantially to the understanding of this field. Administration of aspirin in patients with a distal radius fracture was associated with a lower incidence of CRPS, though not statistically significant. As with other chronic disorders, the future of CRPS prevention and treatment may lie in combination therapy. Considering the limitations of the present study, further investigations needs to be done with a larger sample size, longer followup period and multi-center design.

List of acronyms

AO/OTA - AO-Müller/Orthopaedic Trauma Association ASA - aspirin

CONSORT - Consolidated Standards of Reporting

Trials

CRPS - Complex regional pain syndrome

NSAIDs - Nonsteroidal anti-inflammatory drugs

PLA - placebo

RCT - randomized controlled trial

RSD - Reflex Sympathetic Dystrophy

\section{Authors contributions}

All authors played a substantial role in data acquisition and analysis, and in conception and revision of the manuscript.

\section{Acknowledgments}

The authors wish to thank Rasoul Akram Hospital Clinical Research Development Center (RCRDC) for its technical assistance.

Funding There was no funding support for this study.

\section{Conflict of Interest}

The authors have no conflicts to disclose.

\section{Ethical Publication Statement}

We confirm that we have read the Journal's position on issues involved in ethical publication and affirm that this report is consistent with those guidelines. 


\section{Corresponding Author}

Nima Hosseinzadeh, Bone and Joint Reconstruction Research Center, Shafa Orthopedic Hospital, Iran University of Medical Sciences, Tehran, Iran.

Tel: +98 (21) 64352264

Email: hosseinzade.nima@gmail.com

\section{E-mails of co-authors}

Amir SobhaniEraghi:amir_sobhany@yahoo.com Amir Khazanchin: amir.khazanchin@gmail.com Alireza Pahlevansabagh:Pahlevansabagh@yahoo.com

\section{References}

1. Shah AS, Verma MK, Jebson PJ.Use of oral vitamin $\mathrm{C}$ after fractures of the distal radius. J Hand Surg Am. 2009;34:1736-8.

2. Goh EL, Chidambaram S, Ma D.. Chidambaram, and D. Ma, Complex regional pain syndrome: a recent update. Burns Trauma. 2017;5:2.

3. Harden RN, Bruehl S, Perez RS,et al., Validation of proposed diagnostic criteria (the "Budapest Criteria") for Complex Regional Pain Syndrome. Pain. 2010;150:268-74

4. Bordbar A, Mohagheghi P, Yoonesi L, et al. Value of Physical Examination in the Diagnosis of Developmental Hip Dislocation in Preterm Infants, J Compr Ped. 2018 ; 9:e14049

5. Kashaki M, Masoudi Samghabadi F, Bordbar A. Effect of Fortification of Breast Milk in Conjugation with Protein Supplement on Neurodevelopment of Preterm Low Birth Weight Infants at 3 Years.MEDARCH:2019; 73-5: 344-350

6. Mackey S, Feinberg S. Pharmacologic therapies for complex regional pain syndrome. Curr Pain Headache Rep. 2007;11:38-43.

7. Marinus J, Moseley GL, Birklein F, et al. Clinical features and pathophysiology of complex regional pain syndrome. Lancet Neurol. 2011;10:637-48

8. Zhao Z, Chen SR, Eisenach JC, et al. Spinal cyclooxygenase-2 is involved in development of allodynia after nerve injury in rats. Neuroscience. 2000;97:743-8

9. Syriatowicz JP, $\mathrm{Hu}$ D, Walker JS,et al. Hyperalgesia due to nerve injury: role of prostaglandins. Neuroscience. 1999;94:587-94.

10. Mackey S, Feinberg S. Pharmacologic therapies for complex regional pain syndrome. Curr Pain Headache Rep, 2007: 11: 38-43.

11. Ossipov MH, Jerussi TP, Ren K,et al. Differential effects of spinal (R)-ketoprofen and (S)-ketoprofen against signs of neuropathic pain and tonic nociception: evidence for a novel mechanism of action of (R)-ketoprofen against tactile allodynia. Pain. 2000;87:193-9.

12. Kashaki M, Alipour R, Saboute M. Changes in Vitamin D Status during the First Two Weeks of Life in Preterm Infants on TPN: A Cross Sectional Study. Prensa Med Argent 2019:105:135
13. Lanas A, McCarthy D, Voelker M et al. Short-Term Acetylsalicylic Acid (Aspirin) Use for Pain, Fever, or Colds - Gastrointestinal Adverse Effects. Drugs RD. 2011;11:277-88.

14. Harden RN, Bruehl S, Stanton-Hicks M,et al. Proposed new diagnostic criteria for complex regional pain syndrome. Pain Med. 2007;8:326-31.

15. Sebastin SJ. Complex regional pain syndrome. Indian J Plast Surg. 2011; 44: 298-307.

16. Bruehl S, Chung OY. Psychological and behavioral aspects of complex regional pain syndrome management. Clinic J Pain, 2006; 22: 430-437.

17. Crijns TJ, van der Gronde BATD, et al. Complex regional pain syndrome after distal radius fracture is uncommon and is often associated with fibromyalgia. Clin Orthop Relat Res. 2018 ;476:744-750.

18. Pepper A, Li W, Kingery WS, et al. Changes resembling complex regional pain syndrome following surgery and immobilization. J Pain. 2013;14:516-24

19. Zollinger PE, Tuinebreijer WE, Breederveld RS,et al. Can vitamin $\mathrm{C}$ prevent complex regional pain syndrome in patients with wrist fractures?: A randomized, controlled, multicenter dose-response study. J Bone Joint Surg Am. 2007;89:1424-31.

20. Atkins RM. Principles of complex regional pain syndrome. Fractures in Adults. 7th edn. Philadelphia: Lippincott Williams \& Wilkins, 2011.p. 73

21. Demir SE, Ozaras N, Karamehmetoğlu SS,et al. Risk factors for complex regional pain syndrome in patients with traumatic extremity injury. Ulus Travma Acil Cerrahi Derg. 2010;16:144-8.

22. Roh YH, Lee BK, Noh JH,et al. Factors associated with complex regional pain syndrome type I in patients with surgically treated distal radius fracture. Arch Orthop Trauma Surg, 2014; 134: 1775-81.

23. Ortiz-Romero J, Bermudez-Soto I, TorresGonzález R, et al. Factors associated with complex regional pain syndrome in surgically treated distal radius fracture. Acta Ortop Bras, 2017:25: 194-196.

24. Jellad A, Salah S, Ben Salah Frih Z.Complex regional pain syndrome type I: incidence and risk factors in patients with fracture of the distal radius. Arch Phys Med Rehabil. 2014;95:487-92.

25. Jo YH, Kim K, Lee BG,et al. Incidence of and Risk Factors for Complex Regional Pain Syndrome Type 1 after Surgery for Distal Radius Fractures: A Population-based Study. Sci Rep. 2019;9:4871.

26. de Mos M, Huygen FJ, Dieleman JP, et al. Medical history and the onset of complex regional pain syndrome (CRPS). Pain. 2008;139:458-66.

27. Chung KC, Mathews AL. Management of Complications of Distal Radius Fractures. Hand clinics, 2015:31: 205-215. 


\section{Complex regional pain syndrome}

Eur J Trans1 Myol 30 (1): xx1-xx8, 2020

28. Zyluk, A. Complex regional pain syndrome type I. Risk factors, prevention and risk of recurrence. J Hand Surg Br, 2004:29: 334-7.

29. Cao Y, Xiong J, Mei S, et al. Aspirin promotes bone marrow mesenchymal stem cell-based calvarial bone regeneration in mini swine. Stem Cell Res Ther, 2015;6: 210.

30. Du M, Pan W, Yang P, et al. [Effect of aspirin on cell biological activities in murine bone marrow stromal cells]. Zhonghua Kou Qiang Yi Xue Za Zhi, 2016. 51: 160-5

31. Chin KY. A Review on the Relationship between Aspirin and Bone Health. J Osteoporos 2017. 2017: 3710959.

32. Carbone LD, Tylavsky FA, Cauley JA,et al. Association between bone mineral density and the use of nonsteroidal anti-inflammatory drugs and aspirin: impact of cyclooxygenase selectivity. J Bone Miner Res, 2003;18: 1795-802.

33. Lane NE, Bauer DC, Nevitt MC, et al. Aspirin and nonsteroidal antiinflammatory drug use in elderly women: effects on a marker of bone resorption. The Study of Osteoporotic Fractures Research Group. J Rheumatol, 1997;24: 1132-6.

34. Bauer DC, Orwoll ES, Fox KMet al. Aspirin and NSAID use in older women: effect on bone mineral density and fracture risk. Study of Osteoporotic Fractures Research Group. J Bone Miner Res, 1996;11: 29-35.

35. Lack WD, Fredericks D, Petersen E,et al. Effect of aspirin on bone healing in a rabbit ulnar osteotomy model. J Bone Joint Surg Am. 2013 20;95:488-96.

36. David Clark J, Tawfik VL, Tajerian M,et al., Autoinflammatory and autoimmune contributions to complex regional pain syndrome. Mol Pain. 2018; 14:1744806918799127. doi: 10.1177/1744806918799127. Epub 2018 Aug 20.

37. van der Laan L, ter Laak HJ, Gabreëls-Festen A,et al. Complex regional pain syndrome type I (RSD): pathology of skeletal muscle and peripheral nerve. Neurology. 1998 ;51:20-5.

38. van der Laan L, Oyen WJ, Verhofstad AA, et al. Soft tissue repair capacity after oxygen-derived free radical-induced damage in one hindlimb of the rat. J Surg Res. 1997;72:60-9.

39. Atkins, RM. Complex Regional Pain Syndrome, in Rockwood and Green's Fractures in Adults. 2015:25-28

40. Koseoglus MH, Cuhadar S, Atay A, et al. Effects of Aspirin on Serum Total Antioxidantios] Activity in A Short Term Period. Siriraj Med J 2014;66;3438

41. Demirci B, Demir O, Dost T, et al. Antioxidative effect of aspirin on vascular function of aged ovariectomized rats. Age, 2014;36: 223-229.

42. Podhaisky HP, Abate A, Polte T, et al. Aspirin protects endothelial cells from oxidative stress-possible synergism with vitamin E. FEBS Lett. 1997 ;417:349-51.

43. Ristimäe T, Zilmer M, Zilmer K,et al. Effect of lowdose aspirin on the markers of oxidative stress. Cardiovasc Drugs Ther, 1999;13: 485-90.

44. Kashaki M, Bordbar A, Mazouri A, et al. Determination of the Effect of High-Dose Intralipid in Compared to Its Gradual Dose in Very Low Birth Weight Newborns: A Case-control Study. J Pharmaceutical Res Int 2019:30:1-7.

45. Bordbar A, Hadipour M, Kashaki M. Determination of the Prevalence of Patent Ductus Arteriosus in Infants with Very Low Birth Weight Admitted in Hospital during 2011-2016. Prensa Med Argent 2019:105:134.

Submission: Septamber, 25, 2019

Revision received: October 18, 2019

Acceptance: October 30, 2019 